\title{
Trapped Ion Chain as a Neural Network: Error Resistant Quantum Computation
}

\author{
Marisa Pons ${ }^{1}$, Veronica Ahufinger ${ }^{2}$, Christof Wunderlich ${ }^{3}$, Anna Sanpera ${ }^{4}$, \\ Sibylle Braungardt*, Aditi Sen(De)*, Ujjwal Sen*, and Maciej Lewenstein ${ }^{5}$ \\ ${ }^{1}$ Departamento de Física Aplicada I, Universidad del País Vasco, 20600 Eibar, Spain. \\ ${ }^{2}$ ICREA and Grup d'Òptica, Departament de Física, \\ Universitat Autònoma de Barcelona, 08193 Bellaterra (Barcelona), Spain. \\ ${ }^{3}$ Fachbereich Physik, Universität Siegen, 57068 Siegen, Germany. \\ ${ }^{4}$ ICREA and Grup de Física Teòrica, Departament de Física, \\ Universitat Autònoma de Barcelona, 08193 Bellaterra (Barcelona), Spain. \\ ${ }^{5}$ ICREA and ${ }^{*}$ ICFO-Institut de Ciències Fotòniques, 08860 Castelldefels (Barcelona), Spain.
}

\begin{abstract}
We demonstrate the possibility of realizing a neural network in a chain of trapped ions with induced long range interactions. Such models permit one to store information distributed over the whole system. The storage capacity of such network, which depends on the phonon spectrum of the system, can be controlled by changing the external trapping potential. We analyze the implementation of error resistant universal quantum information processing in such systems.
\end{abstract}

The Cirac-Zoller proposal of trapped ion computer [1] has become one of the paradigmatic models to implement a quantum computer [2]. Recently, spectacular experimental progress in realization of simple algorithms and implementation of quantum logic has been achieved using a few ionic qubits (c.f. [3] ). Although the achievement of an all-purpose quantum computer in the near future seems difficult, one can be quite optimistic about the applications of chains of trapped ions as quantum simulators. Recently, it has been shown that long range (LR) pairwise interactions between individual spins are induced in an ion trap, when applying an additional state-dependent force acting on the ions [4, 5]. Also a state-dependent optical force can evoke LR couplings and has been proposed to simulate spin $1 / 2$ chain systems [6]

Here we show that ion spin systems can serve to realize a neural network (NN) model. $\mathrm{NN}$ are a prototype model of parallel distributed memory [7, 8], and have been intensively studied by physicists since the famous paper by Hopfield [9]. These disordered systems with LR interactions, typically present a large number of metastable (free) energy minima, like in spin glasses 8]. These states can be used to store information distributed over the whole system. The patterns stored have large basins of attraction in the thermodynamical sense, so that even fuzzy ones are recognized as perfect ones. For this reason, attractive NN's can be used as associative memory. At the same time, NN are robust, so that destroying even a large part of the network does not necessarily diminish its performance. The above listed properties make NN's interesting for distributed quantum information (QI), where quantum bits do not correspond to the internal states (spins) of individual ions, but to patterns of the internal states of the whole chain (all-up, all-down, half-up-half-down, etc.). These patterns echo the lowest energy vibrational modes of the system. The sign of the displacement of each ion with respect to its equilibrium position in a given mode fixes the up/down state of the spin in the associated spin pattern. Some approaches to exploit the potential of NN models for QI processing have been discussed [10], also with respect to entanglement generation [11]. Here we propose, for the first time, a feasible implementation of NN, and the realization of distributed QI using a chain of trapped ions.

We first remind the readers the main features of the Hopfield model [9], and discuss its similarities with the effective Hamiltonian derived in Ref. [5, 6], that suggest the possibility of using a chain of trapped ions as a NN. We find the ion-chain storage capacity and its robustness the most appealing features of $\mathrm{NN}$ for distributed QI. Thus, the question of ergodicity and, therefore, the ability of the system to act as an associative memory is not considered here. We show that the storage capacity, which is determined by the phonon spectrum of the system, can be controlled by modifying the shape of the external axial trapping potential and/or by applying longitudinal magnetic fields. Although this is a classical property of the network, spin ion systems also permit to study quantum $\mathrm{NN}$ by applying a transverse magnetic field or an optical field that effectively simulates it. Here, we exploit the storage capacity of the system to perform distributed QI i.e. single and two-qubit gates by applying appropriate external axial and transverse magnetic fields. Transverse magnetic fields should also permit tunneling processes between stored patterns and to realize, for example, quantum stimulated annealing [8, 12]. This study is beyond the scope of this Letter and will be treated elsewhere.

Following the models of Hopfield [9] and Little [13], a neuron can be viewed as an Ising spin with two possible states: "up" $(S=+1)$ and "down" $(S=-1)$ depending on whether the neuron has or has not fired an electromagnetic signal, in a given interval of time 7]. The state of the network of $N$ neurons at a certain time is defined by the instantaneous configuration of all the spins $\left\{S_{i}\right\}$ at this time. The dynamic evolution of these states is determined by the symmetric interactions among neurons, $J_{i j}=J_{j i}$. Also, full connectivity is assumed, i.e., every 
neuron can receive an input from any other one, and send an output to it. The Hamiltonian reads:

$$
H=-\frac{1}{2} \sum_{i, j}^{N} J_{i j} S_{i} S_{j}+h \sum_{i}^{N} S_{i},
$$

where $h$ corresponds to an external magnetic field. The interactions are determined by the patterns or configurations of spins to be stored in the network. These patterns will be learned if the system is able to accommodate them as attractors, implying that a large set of initial configurations of the network will be driven dynamically to those patterns. A possible choice of the interactions is

$$
J_{i j}=\frac{1}{N} \sum_{\mu=1}^{p} \xi_{i}^{\mu} \xi_{j}^{\mu},
$$

with $i \neq j$. The $p$ sets of $\left\{\xi_{i}^{\mu}\right\}= \pm 1$ are the patterns to be stored. The network will have the capacity of storage and retrieval of information, if the dynamical stable configurations (local minima) reached by the system $\left\{S_{i}\right\}$ are correlated with the learned ones $\left\{\xi_{i}^{\mu}\right\}$. Although the interactions have been constructed to guarantee that certain specified patterns are fixed points of the dynamics, the non-linearity of the dynamical process may induce additional attractors, the so-called spurious states.

Recently it has been shown that the Hamiltonian describing a linear chain of harmonically trapped ions exposed to a magnetic field gradient [5] or interacting with convenient laser fields [ 6 ] can be transformed into an effective spin-spin hamiltonian with LR interactions $\left(J_{i j}^{\alpha}\right)$, mediated by the collective motion of the ions:

$$
\begin{gathered}
H=-\frac{1}{2} \sum_{\alpha, i, j} J_{i j}^{\alpha} \sigma_{i}^{\alpha} \sigma_{j}^{\alpha}+\sum_{\alpha, i} B_{i}^{\alpha} \sigma_{i}^{\alpha}, \\
J_{i j}^{\alpha}=\frac{\left(F^{\alpha}\right)^{2}}{m} \sum_{n} \frac{M_{i, n}^{\alpha} M_{j, n}^{\alpha}}{\omega_{\alpha, n}^{2}},
\end{gathered}
$$

with $\alpha=x, y, z,(i, j)$ label the ions, $\sigma$ are the Pauli matrices, $F^{\alpha}$ the force in the $\alpha$ direction experimented by the ions, $m$ the ion mass and $\omega_{\alpha, n}$ the angular frequency of the vibrational mode $n . M_{i, n}^{\alpha}$ are the unitary matrices that diagonalize the vibrational Hamiltonian: $M_{i, n}^{\alpha} \kappa_{i, j}^{\alpha} M_{j, m}^{\alpha}=\omega_{\alpha, n}^{2} \delta_{n m}$, where $\kappa_{i, j}^{\alpha}$ are the elastic constants of the chain [14]. The coefficient $M_{i, n}^{\alpha}$ gives the scaled amplitude of the local oscillations of ion $i$ around its equilibrium position, when the collective vibrational mode $n$ is excited. Thus, the eigenvectors of $M$ describe each ion's contribution to a given vibrational mode, while the eigenvalues provide the frequencies, $\omega_{\alpha, n}$ of the collective modes.

The external trapping frequencies are chosen such that the laser cooled ions crystallize in a linear chain (i.e. $\left.\omega_{x, 1}=\omega_{y, 1} \gg \omega_{z, 1}\right)$ and the external forces act on the $z$-axis (i.e. $F^{x}=F^{y}=0$ ), so that the index $\alpha$ is dropped. Henceforth, we consider zero magnetic fields $B_{i}=0$ [15]. If we substitute the Pauli matrices in Eq.(3) by Ising spins $S= \pm 1$ (where the effective spin corresponds to the internal state of the ion), we recover Eq.(1) and the possibility to implement a classical NN with this system arises. Nevertheless, there are some differences between both models. First, in the Hopfield model, the interactions (Eq.(2)) are determined by the patterns to be stored $\left\{\xi_{i}^{\mu}\right\}= \pm 1$, while in the trapped ion chain, the interactions are fixed by the collective modes of the system, i.e., the coefficients $M_{i, n}$ that do not necessarily equal \pm 1 . Second, in Eq.(2), $p$ corresponds to the number of patterns to be stored, which in the limit of large $N$ (number of spins), is bounded from above by $p=0.14 \mathrm{~N}$ [7. In Eq.(44), the sum extends over the total number of vibrational modes which is larger than the total number of stored patterns (spin configurations that the system is able to recover). And finally, in the Hopfield model all the patterns have the same weight while in the ion chain each vibrational mode is weighted by $1 / \omega_{n}^{2}$ (Eq.(4)). To reproduce as closely as possible a NN behavior, the most relevant requirement is the degeneracy of the vibrational modes. Moreover, the corresponding patterns must have large basins of attraction, i.e. they should correspond to sufficiently different spin configurations, so that each one is dynamically recovered, even if several spins are randomly flipped.

To check the feasibility of implementing a NN model in these ion spin systems, we first find the phonon spectrum using a standard diagonalization procedure, and impose the learning rule i.e., we calculate the spin interactions $J_{i j}$, mediated by the collective modes of the ions (Eq.(44)). Then, we map a given vibrational mode into a spin configuration (initial spin configuration), evaluate its energy (Eq.(3)) and check its dynamical stability under spin flips using a standard Metropolis algorithm in a classical Monte Carlo code. This stability is essential for adiabatic QI processing. Explicitly, from the initial configuration we randomly flip $r$ spins, and let the system evolve towards equilibrium assuming a noiseless scenario. If the system recovers the initial configuration, it is stable under the flip of $r$ spins. We define the initial overlap as $m_{i}=(N-r) / N$. After dynamical evolution, the final overlap is given by $m_{j}=(N-s) / N$ where $s$ is the number of spins that differ from the initial configuration. We repeat this process over $M$ initial configurations each with $r$ random spin flips. We evaluate statistically the final overlap with the initial configuration as: $m_{f}=\left(\sum_{j=1}^{N} m_{j} n_{j}\right) / M$, being $n_{j}$ the number of times that the system reaches the configuration with $m_{j}$. The value of $m_{i}$ for which significant decrease of $m_{f}$ occurs, is a good measure of the size of the pattern's basin of attraction. For the harmonic trapping potential, the two lowest vibrational modes are the center of mass (CM) (all spins parallel, with $\omega_{1}$ ) and the breathing (B) 

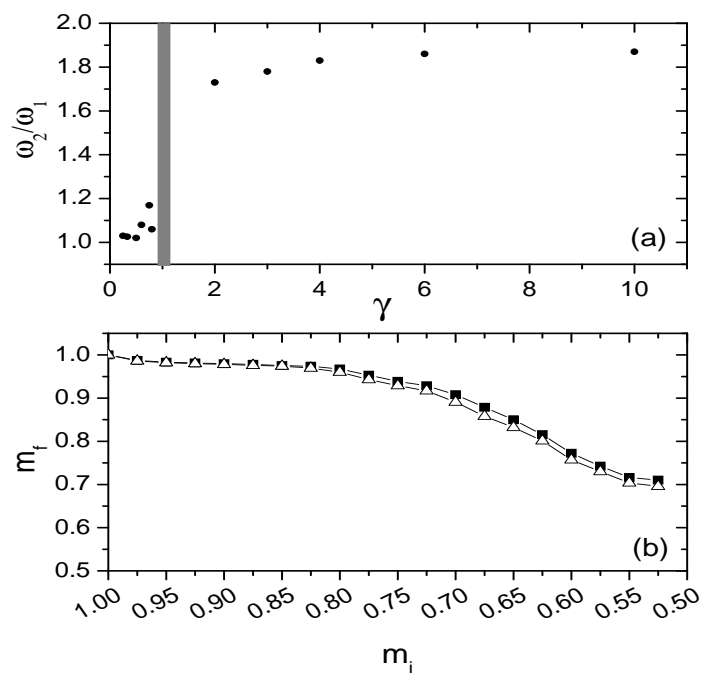

FIG. 1: (a) Ratio between the frequencies of the second and first vibrational modes as a function of the exponent of the trapping potential for $20 \mathrm{Ca}^{+}$ions. (b) Final overlap averaged over 500 initial configurations as a function of the initial overlap, for the patterns associated with the two lowest vibrational modes of 40 ions in a potential $V=\rho|x|^{0.5}$ with $\rho=6.6 \times 10^{-20} \mathrm{~J} / \mathrm{m}^{1 / 2}$. The black squares (triangles) correspond to the first (second) pattern.

mode (half up, half down, with $\omega_{2}=\sqrt{3} \omega_{1}$ ) [16]. We find that the pattern associated with the CM mode is stable up to the flip of half of the spins, while the one associated with the B mode is already unstable under a single spin flip. Thus, only the spin configuration associated with the CM mode can be recovered (i.e., stored). To increase the storage capacity of the network, we consider here $V(x)=\rho|x|^{\gamma}$, achievable using additional control by dc electrodes ([4](b), see also [17]). We calculate the ratio $\omega_{2} / \omega_{1}$, as a function of $\gamma$, for $\mathrm{Ca}^{+}$ions. For $N \geq 20$, this ratio depends neither on the number of ions, nor on the value of $\rho$. For $\gamma \geq 1$, the ratio is $\approx \sqrt{3}$, and as in the harmonic case, severe limitations on the storage capacity appear (see Fig.1(a)). However, for $0.25<\gamma<0.8$, the two lowest modes become nearly degenerate. The storage capacity for a system of $40 \mathrm{Ca}^{+}$ ions trapped in a potential with $\gamma=0.5$ is displayed in Fig. 1(b), where the final overlap is depicted as a function of the initial overlap for the patterns associated with the two lowest vibrational modes. $m_{f}$ is close to 1 up to 8 spin flips, meaning that the system is able to recover four patterns (the two associated with the two lowest modes plus the two corresponding to a global spin flip). The system sometimes reaches a slightly deformed configuration, which differs only in 1 spin flip from the original one (spurious states), making $m_{f}$ slightly smaller than 1 . Specifically, the probability of recovering the two modes is above $98 \%$, up to 3 initial random spin flips, and above $97 \%$ up to $8\left(m_{i}=0.8\right)$.

Having shown that our system can be robust for clas-
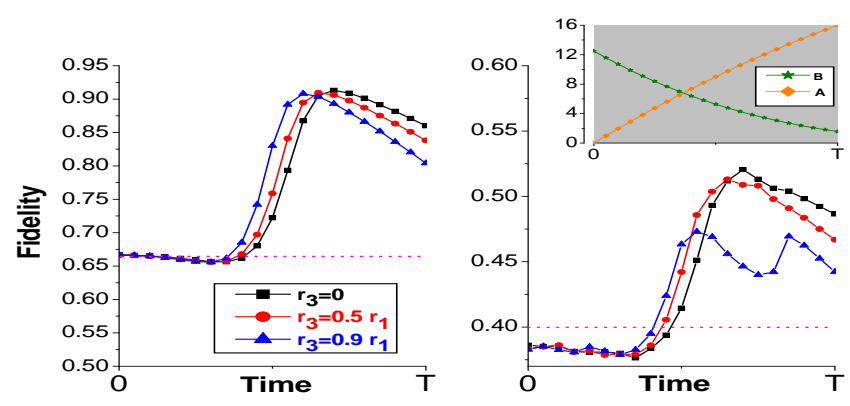

FIG. 2: (Color online) Fidelities of the $\mathcal{H}$ gate (left) and the Bell gate (right), with respect to time $\left(r_{2}=0.95 r_{1}\right)$. The classical fidelity bounds are $2 / 3$ and $2 / 5$, respectively (horizontal dashed lines). The inset in gray shows the fields $A \lambda$ and $B_{1} \lambda=10^{-5} B \lambda$ and $B_{2} \lambda=10^{-6} B \lambda$ with respect to time. For adiabaticity, the chosen fields require $T \gg 7 \times 10^{6} \hbar / \lambda$. The fidelities are largely independent of the actual dynamics of the fields.

sical information storage, we explore now its capability for distributed quantum computing in an error resistant way (cf. [18]), i.e. robust with respect to the partial damage of the system. To this aim, we consider a system of 8 spin- $1 / 2$ particles (as in [19]) in a trapping potential with $\gamma \simeq 0.5$. The vibrational spectrum is, except for the lowest two modes, highly non-degenerate, with rapidly increasing eigenvalues. Thus, we consider only the contributions of the spin configurations associated with the three lowest motional modes which, up to a gauge transformation, correspond to: all-up, half-up-half-down, 2up-4-down-2-up. We encode the information in the first two, and consider the third one as noise. The phonon mode amplitudes are approximated as $M_{i, m}=\xi_{i}^{m}= \pm 1$ (exact for periodic boundary conditions). Additionally, time dependent "magnetic" fields in the $z\left(B_{1}, B_{2}\right)$ and $x$ $(A)$ directions are applied leading to the following expression for a Quantum Neural Network (QNN) Hamiltonian:

$$
\begin{aligned}
& H_{Q N N}(t)=-\lambda\left[r_{1}\left(S_{1}^{z}+S_{2}^{z}+S_{3}^{z}+S_{4}^{z}\right)^{2}+\right. \\
& r_{2}\left(S_{1}^{z}+S_{2}^{z}-S_{3}^{z}-S_{4}^{z}\right)^{2}+r_{3}\left(S_{1}^{z}-S_{2}^{z}-S_{3}^{z}+S_{4}^{z}\right)^{2} \\
& +A\left(S_{1}^{x}+S_{2}^{x}+S_{3}^{x}+S_{4}^{x}\right) \\
& \left.+B_{1}\left(S_{1}^{z}+S_{2}^{z}\right)+B_{2}\left(S_{3}^{z}+S_{4}^{z}\right)\right]
\end{aligned}
$$

where $S_{i}^{\alpha}=\sigma_{2 i-1}^{\alpha}+\sigma_{2 i}^{\alpha}, i=1, \ldots, 4$, and $r_{1} \approx r_{2} \gg r_{3}>$ 0 . With $\lambda$ in energy units, all the other parameters in $H_{Q N N}$ are dimensionless. $B_{1}, B_{2}$, and $A$ are chosen initially $\left(t=t_{0}\right)$ such that the ground $\left|G\left(t_{0}\right)\right\rangle$, and the first three excited states $\left|E_{j}\left(t_{0}\right)\right\rangle$ correspond to: $|\uparrow \uparrow \uparrow \uparrow \uparrow \uparrow \uparrow \uparrow\rangle_{z}$, $|\downarrow \downarrow \downarrow \downarrow \downarrow \downarrow \downarrow \downarrow\rangle_{z},|\uparrow \uparrow \uparrow \uparrow \uparrow \downarrow \downarrow \downarrow \downarrow\rangle_{z}$, and $|\downarrow \downarrow \downarrow \downarrow \uparrow \uparrow \uparrow \uparrow\rangle_{z}$.

To demonstrate universality we focus first on a single qubit operation. Identifying $|0\rangle=\left|G\left(t_{0}\right)\right\rangle,|1\rangle=\left|E_{1}\left(t_{0}\right)\right\rangle$, we consider a single distributed-qubit operation, with a generic qubit $a_{0}\left|G\left(t_{0}\right)\right\rangle+a_{1}\left|E_{1}\left(t_{0}\right)\right\rangle$, evolving adiabatically under the changes of the magnetic fields, to a final state $a_{0}|G(t=T)\rangle+a_{1}\left|E_{1}(t=T)\right\rangle$. We choose the time 
dependence of the fields so that the final state is approximately the $\mathcal{H}$-rotated state of the input one, where $\mathcal{H}$ acts on the logical states as $|0\rangle \rightarrow|+\rangle,|1\rangle \rightarrow-|-\rangle$, where $| \pm\rangle=(|0\rangle \pm|1\rangle) / \sqrt{2}$. This one-qubit gate $\mathcal{H}$ is reminiscent of the Hadamard gate that takes $|0\rangle \rightarrow|+\rangle$, $|1\rangle \rightarrow|-\rangle$. The $\mathcal{H}$ operation is achieved by changing the positive initial values of $B_{1}$ and $B_{2}$ adiabatically to zero, and simultaneously increasing the zero initial $A$ to a positive value much larger than the initial $B_{1}, B_{2}$ (inset Fig. 2). Since we deal with superpositions of energy eigenstates, we consider in the adiabatic transport the dynamical, as well as the Berry phases [20]. The fidelity of the $\mathcal{H}$ gate is shown in Fig. 2(left) as a function of time for different noise ratio $r_{3} / r_{1}$. Note that artificially increasing the ratio $r_{3} / r_{1}$ imitates inaccuracies in the trapping potential, disturbance in the motion of the ions, as well as noise in the spin (as the phonons are the carriers of interaction between the spins). The fidelity is quite insensitive to high noise levels, and is larger than the classical (measure and prepare) bound of $2 / 3$.

Let us move now to the two-qubit gates, and treat the 4 left spins as one qubit, and 4 right ones as another, so that: $|00\rangle=|\uparrow \uparrow \uparrow \uparrow \uparrow \uparrow \uparrow \uparrow \uparrow\rangle_{z},|01\rangle=|\uparrow \uparrow \uparrow \uparrow \downarrow \downarrow \downarrow \downarrow\rangle_{z}$, etc. (We have checked that the $\mathcal{H}$ gate fidelity is robust in this encoding also.) We demonstrate here, a way to implement an entangling universal gate [21] acting as $|00\rangle \rightarrow(|00\rangle+|11\rangle) / \sqrt{2},|11\rangle \rightarrow(-|00\rangle+|11\rangle) / \sqrt{2}$, $|01\rangle \rightarrow(|01\rangle+|10\rangle) / \sqrt{2},|10\rangle \rightarrow(-|01\rangle+|10\rangle) / \sqrt{2}$. We denote this gate as Bell gate. We now encode an arbitrary two-qubit state $\sum_{i, j=0,1} a_{i j}|i j\rangle$, into $a_{00}\left|G\left(t_{0}\right)\right\rangle+$ $a_{11}\left|E_{1}\left(t_{0}\right)\right\rangle+a_{01}\left|E_{2}\left(t_{0}\right)\right\rangle+a_{10}\left|E_{3}\left(t_{0}\right)\right\rangle$. The same variation of the magnetic fields as in the $\mathcal{H}$ gate leads now to the Bell-gate rotated state (Fig. 2 (right)), with fidelity that is noise insensitive and surpasses the classical bound of $2 / 5$. Note that in addition to being resistant against noise induced by increasing $r_{3} / r_{1}$, the fidelities are also robust against spin-flip errors, as we have encoded the qubit(s) in the two (four) lowest energy levels, for the $\mathcal{H}$ (Bell) gate, which we have already shown to be metastable against spin-flips. The time scales for which both $\mathcal{H}$ and Bell gate fidelities reach maximum values are long enough to ensure robust implementation and also robustness against errors in time of observation.

Summarizing, we have shown that spin-ion systems can be used to implement NN models. We have calculated their storage capacity and robustness against spin flips as well as their dependence on the trapping potential. Identifying the qubits with configurations of spins that echo the lowest vibrational modes of the system, we have shown that the system can perform error resistant universal distributed QI processing. We have demonstrated that by applying adiabatically-varying time dependent "magnetic" fields, the system realizes single and two distributed-qubit operations in a robust way [22]. The scalability issue is like other proposals and experiments in ion-trap quantum computing [23], and may po- tentially be overcome by connecting mesoscopic clusters of trapped ions for instance, by "flying" qubits.

We thank I. Bloch, A. Bramon, H.-P. Büchler, J. Eschner, M. Mitchell, J. Wehr, P. Zoller for fruitful discussions, and acknowledge German DFG SFB 407, ESF PESC QUDEDIS, Spanish MEC (FIS200504627;FIS2005-01369, Consolider-Ingenio2010 CSD200600019), AvH Foundation, EU IP SCALA and QAP for support.

[1] J.I. Cirac and P. Zoller, Phys. Rev. Lett. 74, 4091 (1995).

[2] D. Bouwmeester, A. Ekert, and A. Zeilinger (Eds.), The Physics of Quantum Information (Springer, Berlin, 2000).

[3] F. Schmidt-Kaler et al., Nature 422, 408 (2003); J. Chiaverini et al., Science 308, 997 (2005); M. Riebe et al., Nature 429, 734 (2004); M. D. Barrett et al, Nature 429, 737 (2004).

[4] (a) F. Mintert and C. Wunderlich, Phys. Rev. Lett. 87, 257904 (2001); Ch. Wunderlich and Ch. Balzer, Adv. At. Mol. Opt. Phys. 49, 293 (2003); (b) D. McHugh and J. Twamley, Phys. Rev. A 71, 012315 (2005).

[5] C. Wunderlich, in Laser Physics at the Limit (Springer, Heidelberg, 2002); also available as quant-ph/0111158.

[6] D. Porras and J.I. Cirac, Phys. Rev. Lett. 92, 207901 (2004).

[7] D.J. Amit, Modeling Brain Function (Cambridge University Press, Cambridge, 1989).

[8] M. Mézard, G. Parisi, and M.A. Virasoro, Spin Glass Theory and Beyond (World Scientific, Singapore, 1987).

[9] J.J. Hopfield, Proc. Natl. Acad. Sci. 81, 3088 (1984).

[10] P. Gralewicz, quant-ph/0401127, and references therein.

[11] A. Sen(De) et al., Phys. Rev. A 74, 062309(2006).

[12] D.J. Amit, H. Gutfreund and H. Sompolinsky, Phys. Rev. A 32, 1007 (1985).

[13] W.A. Little, Math. Biosci. 19, 101 (1974).

[14] H. Goldstein, Classical Mechanics (Addison-Wesley, MA, 1980).

[15] The effective magnetic field $B$ in Eq. (3) includes a contribution from the applied lasers, which can be compensated by a constant magnetic field $B^{\prime}[\underline{6}]: B=B^{\prime}+\frac{F^{2}}{m \omega_{1}^{2}}$.

[16] D.F.V. James, Appl. Phys. B 66, 181 (1998); G. Morigi and S. Fishman, Phys. Rev. Lett. 93, 170602 (2004).

[17] The storage capacity of the system can be also increased by engineering the spatial dependence of a longitudinal magnetic field.

[18] http://www.perimetertinstitute.ca/personal/dgottesman/ and references therein.

[19] H. Häffner et al., Nature 438, 643 (2005).

[20] A. Shapere and F. Wilczek, Geometric Phases in Physics, (World Scientific, Singapore, 1998).

[21] D. Deutsch, A. Barenco and A. Ekert, Proc. Roy. Soc. London A 449, 669 (1995).

[22] It has certain similarities, but also differences with adiabatic quantum computation. See e.g. E. Farhi and S. Gutmann, Phys. Rev. A 57, 2403 (1998).

[23] See e.g., S. Seidelin et al., quant-ph/0601173. 